\title{
Ophthalmic artery flow velocity in glaucomatous and normal subjects
}

\author{
Prin Rojanapongpun, Stephen M Drance, Brenda J Morrison
}

\begin{abstract}
The velocity of blood flow in the ophthalmic artery was measured with a transcranial Doppler ultrasound (2 MHz). Sixty patients with chronic open angle glaucoma (COAG), 42 patients with normal tension glaucoma (NTG), and 35 normals were studied. Peak flow velocity (peak), mean-enveloped velocity (mean) and diastolic velocity were compared in the three groups. The COAG patients and the NTG patients showed a significant reduction of all three Doppler flow velocities compared with the normals (COAG vs normal, $p=0.013$, 0.019 , and 0.009; NTG vs normal, $p=<0.001$, $<0.001$, and $<0.001$ for peak, mean and diastolic flow velocity respectively). The NTG patients also had a significant slower mean flow velocity than the COAG patients $(p=0.016)$, but not for peak and diastolic flow velocities $(p=0.060$ and 0.052 respectively). Based on ophthalmic flow velocity values, many NTG patients and a few COAG patients had slower flow velocities than the normals. The significance of these haemodynamic differences is not yet known.

(BrF Ophthalmol 1993; 77: 25-29)
\end{abstract}

The mechanisms of glaucomatous damage are still not known. Damage to the optic nerve head can be caused by increased intraocular pressure (IOP) alone. Vascular factors with or without an increase in IOP have been implicated in the development of glaucomatous damage but the mechanisms of their involvement, either as part of generalised vascular disorder or as a purely localised disturbance are still unclear. Many vascular related diseases have been reported to be associated with glaucoma including diabetes, ${ }^{1}$ hypertension, ${ }^{2}$ and migraine. ${ }^{3}$ Peripheral vasospasm was found to be more common in patients with normal tension glaucoma (NTG). ${ }^{4}$ 'Ocular vasospasm' has been proposed as a risk in the pathogenesis of NTG. ${ }^{56}$ The higher prevalence of major vascular crises, including myocardial infarction with shock, major bleeds, and perioperative hypotension in patients with NTG when compared with the age-matched controls,? also suggests that circulatory disturbances may be an associated factor.

One of the more extensive studies of the vascular factors showed no difference in the markers of atherosclerotic vascular disease between patients with chronic open angle glaucoma (COAG), NTG, and non-glaucomatous controls. ${ }^{8}$ The results of the study did not support a generalised organic vascular pathology in glaucoma patients. Direct studies of the ocular circulation in humans remains difficult. Doppler ultrasound has recently been applied to ophthal- mic research. The principle of Doppler ultrasound is well known. ${ }^{9}$ The detection of blood flow velocity is derived from the detection of the ultrasound frequency shift when it is reflected from a multitude of moving blood cells. The transcranial Doppler ultrasound which we used is a $2 \mathrm{MHz}$ pulsed Doppler with a fast Fourier transformation which is used to derive and analyse the spectrum of returning echoes of various frequencies. ${ }^{10}$ It provides discriminated signals from specified depths and well defined regions with little attenuation which is normally produced by the ocular and orbital soft tissues. It has made the non-invasive examination of the ophthalmic artery and some other intracranial vessels possible. " We used this system to measure the blood flow velocity in the ophthalmic artery and have achieved good and acceptable reproducibility. ${ }^{12}$ In this study we compared COAG patients with NTG patients and normal controls.

\section{Subjects and methods}

PATIENTS

We studied 137 subjects consisting of 60 patients with COAG, 42 patients with NTG and 35 subjects with normal eyes (normal). COAG was diagnosed in patients who had characteristic visual field defects and glaucomatous optic neuropathy with multiple IOP readings over $21 \mathrm{~mm} \mathrm{Hg}$. The NTG patients were those whose IOPs were consistently lower than $21 \mathrm{~mm} \mathrm{Hg}$ including diurnal tension studies, and who had the characteristic disc and visual field defects seen in the COAG group. The normals were those who had no ocular abnormality, normal visual fields, and IOPs always below $21 \mathrm{~mm} \mathrm{Hg}$.

\section{Methods}

The ophthalmic artery flow velocity was measured with the transcranial Doppler ultrasound, TCD (2 MHz). We used system MedasonicII Transpect TCD (Medasonics, CA, USA) which had been previously described.$^{12}$ In brief, the patients were tested in the supine position and were instructed to fix their gaze mostly downwards and nasally. The TCD probe was placed on the upper eyelid usually in the superotemporal region and pointed towards the orbital apex. The probe and the patients' eyes were adjusted as to position, direction, and angulation to obtain the best and consistent signals. The power level was set at $10 \%$ which is equivalent to $16 \cdot 1 \mathrm{~mW} / \mathrm{cm}^{2}$ and is well within the safety limit recommended by FDA. ${ }^{13}$ The gated depth was usually set at $\mathbf{4 0} \mathrm{mm}$ and the volume sampled was 7-13 mm long. Peak velocity (peak), 
mean-enveloped velocity (mean), and diastolic velocity in $\mathrm{cm} / \mathrm{s}$ were repeatedly measured in each eye. Peak velocity is defined as the maximum systolic peak velocity. The mean-E velocity is defined as the mean of the peak frequency envelope which outlines all the frequency peaks forming a single signal in one cardiac cycle. Diastolic flow velocity is the minimum flow velocity at the end of the diastolic phase before the next cardiac cycle. All tests were performed by one of us (PR). Demographic information and blood pressure were also recorded. The pulse rate was calculated from the videographic printout.

\section{STATISTICAL ANALYSIS}

Only one eye (the right eye) was selected for analysis to avoid underestimating the replicating error which might occur as a result of the correlation between the two eyes of a person. To compare the three groups, we performed analyses to covariance of all Doppler parameters using diagnostic group as the main factor and age and sex as covariates. The first step was to test the homogeneity of the slopes of the covariates to ensure there was no significant difference in these slopes among the three diagnostic groups. ${ }^{14}$ This was done for each of the two covariates by including terms for the interaction of the covariates with the diagnostic grouping. No significant differences in the slopes of the diagnostic groups were detected and the analyses were therefore repeated omitting these interaction terms. The Bonferroni pairwise post hoc procedure was used to test the differences between the adjusted group means. To determine the comparability of the three groups with regard to other variables which might have an effect on Doppler flow velocity, analyses of variance were performed for age, systolic blood pressure, diastolic blood pressure, and pulse rate. For the analyses of systolic and diastolic blood pressure, age was considered as covariate. Standard errors of means were reported as the number of cases that were different in the three

Table 1 Demographic data of the normal chronic open angle glaucoma, and normal tension glaucoma groups

\begin{tabular}{|c|c|c|c|}
\hline & Normal & $\begin{array}{l}\text { Chronic open } \\
\text { angle glaucoma }\end{array}$ & $\begin{array}{l}\text { Normal tension } \\
\text { glaucoma }\end{array}$ \\
\hline $\begin{array}{l}\text { Numbers (cases) } \\
\text { Sex (female : male) } \\
\text { Age (years) } \\
\text { Age range (years) } \\
\text { Systolic blood pressure }(\text { (mm Hg) } \\
\text { Diastolic blood pressure }(\mathrm{mm} \mathrm{Hg}) \\
\text { Pulse rate }\end{array}$ & $\begin{array}{l}35 \\
19: 16 \\
61 \cdot 87(2 \cdot 05) \\
34 \cdot 57-83 \cdot 08 \\
124 \cdot 99(5 \cdot 28) \\
70 \cdot 63(3 \cdot 49) \\
63 \cdot 92(2 \cdot 48)\end{array}$ & $\begin{array}{l}60 \\
28: 32 \\
64 \cdot 26(1 \cdot 68) \\
32 \cdot 04-81 \cdot 19 \\
133 \cdot 58(3 \cdot 85) \\
74 \cdot 55(2 \cdot 55) \\
60 \cdot 13(2 \cdot 07)\end{array}$ & $\begin{array}{l}42 \\
27: 15 \\
65 \cdot 89(1 \cdot 72) \\
29 \cdot 90-81 \cdot 36 \\
138 \cdot 21(4 \cdot 21) \\
79 \cdot 12(2 \cdot 78) \\
60 \cdot 84(2 \cdot 30)\end{array}$ \\
\hline
\end{tabular}

^Values given are least squares means (standard errors), no statistical difference between groups; tvalues given are adjusted least squares means (standard errors), using age as covariate. No statistical significance between groups. groups. All $p$ values reported in this study were from two-tailed test.

\section{Results}

The demographic and systemic clinical background of the three diagnostic groups is shown in Table 1 . There were no statistically significant differences in pulse rate, systolic, and diastolic blood pressure among the groups at the time of the test $(p=0 \cdot 485,0 \cdot 176$, and $0 \cdot 163$ respectively). The mean ages of the groups were comparable $(p=0 \cdot 358)$. We found a significant effect of age on the diastolic flow velocity in the 137 subjects $(\mathrm{p}=<0.001)$ and a slight effect on peak flow velocity although it was not statistically significant $(p=0.097)$, but not on the mean velocity $(p=0 \cdot 132)$. The sex ratios differed among the groups but analysis of covariance did not demonstrate a statistically significant effect of sex on peak and mean flow velocities $(p=0.084$ and $0 \cdot 167$ respectively) except for diastolic flow velocity and only in the normal group $(p=0.016$, mean (standard error) of female $=10.68(0.78)$ and male $=13.59(0.83) \mathrm{cm} / \mathrm{s})$.

Comparisons were made for each of the three ultrasound measurement between the normals, the COAG, and NTG patients (Table 2). The mean flow velocity value of one eye in the COAG group was not recorded. There was also one, two, and one diastolic flow velocity value missing in the COAG, NTG, and normal groups respectively. The mean peak velocity was lowest in the NTG patients and highest in the normals with a difference in the adjusted means of $8.50 \mathrm{~cm} / \mathrm{s}$ $(p=<0.001)$. The age adjusted mean velocities were lower by $19 \cdot 40 \%$ for peak, $22 \cdot 34 \%$ for mean, and $24.66 \%$ for diastolic velocity in the NTG patients compared with normals. The mean and diastolic velocities were lowest in the NTG group and highly statistically significantly different from the normal group (both $p=<0.001)$. Figure 1 shows the dot density plots of peak flow velocity in the three groups. Mean and diastolic flow velocity distributions are shown in Figures 2 and 3 respectively.

The peak, mean, and diastolic velocities of the COAG patients were also compared with the normals. All differences were statistically significant $(p=0.013,0.019$, and 0.009 respectively). Comparing all flow velocities of the COAG group and NTG group showed statistically significant difference for mean flow velocity $(p=0.016)$. Peak and diastolic flow velocities showed some differences but these were not statistically significant (Table 2).

We identified flow velocity values which were 1.5 and 2.0 standard deviations lower than the mean of the normals as shown by two dotted lines

Table 2 Ophthalmic flow velocity in the normal chronic open angle glaucoma and normal tension glaucoma groups (cm/s, values given in age adjusted means (standard errors))

\begin{tabular}{lllllll}
\hline $\begin{array}{l}\text { Doppler } \\
\text { parameter }\end{array}$ & Normal & $\begin{array}{l}\text { Chronic open } \\
\text { angle } \\
\text { glaucoma }\end{array}$ & $\begin{array}{l}\text { pValue } \\
\text { (two-tailed) }\end{array}$ & $\begin{array}{l}\text { Normal } \\
\text { tension } \\
\text { glaucoma }\end{array}$ & $\begin{array}{l}p \text { Valuet } \\
\text { (two-tailed) }\end{array}$ & $\begin{array}{l}p \text { Valuef } \\
\text { (two tailed })\end{array}$ \\
\hline Peak flow velocity & $43.86(1.32)$ & $39.04(1.00)$ & 0.013 & $35.35(1.20)$ & $<0.001$ & 0.060 \\
Mean flow velocity & $22.87(0.74)$ & $20.28(0.57)$ & 0.019 & $17.76(0.68)$ & $<0.001$ & 0.016 \\
Diastolic flow velocity & $11.92(0.44)$ & $10.25(0.33)$ & 0.009 & $8.96(0.41)$ & $<0.001$ & 0.052
\end{tabular}

*Comparison between the normal and the chronic open angle glaucoma; + Comparison between the normal and the normal tension glaucoma; $¥$ Comparison between COAG and normal tension glaucoma. 
Figure 1 A dot density plot of the right ophthalmic artery peak flow velocity $(\mathrm{cm} / \mathrm{s})$ of 60 chronic open angle glaucoma, 42 normal tension glaucoma patients, 35 normals. Each dot represents the velocity of an eye. The means and standard deviations for the normals are shown: 1.5 and 2.0 standard deviations $(32 \cdot 13$ and $28.22 \mathrm{~cm} / \mathrm{s}$ respectively) from the mean of the normal eye are marked by the dotted lines.

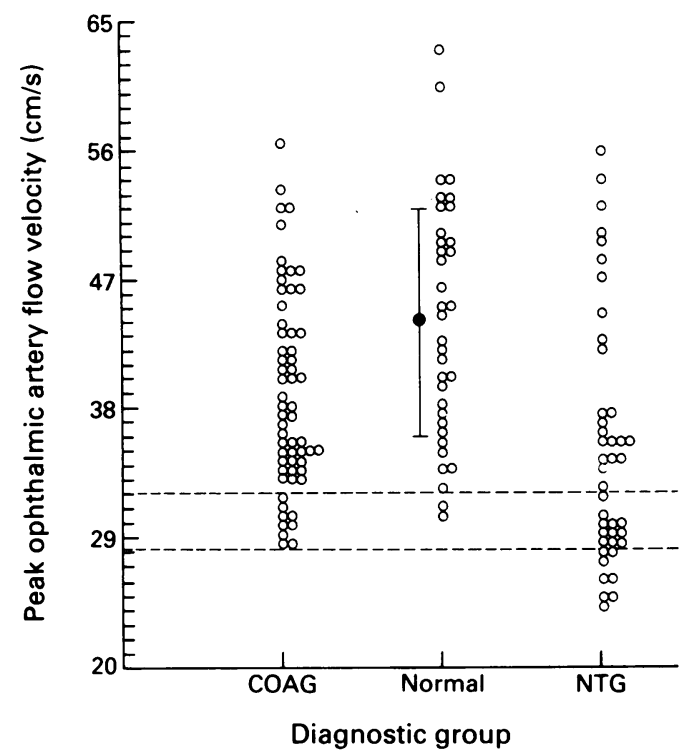

in Figure 1-3. Figure 4 illustrates the percentages of subjects in each group whose peak flow velocity values were 1.5 and $2.0 \mathrm{SD}$ below the normal means. Using the $2.0 \mathrm{SD}$ cut off, $19.05 \%$ of the NTG patients fell outside the range while none of the COAG patients or the normals did. Nearly half of the NTG patients were below the 1.5 SD cut off while $13.33 \%$ of the COAG patients and only $5 \cdot 71 \%$ of normals were below the cut off. Similar findings were seen in mean flow velocity (Figure 5) and to a lesser degree in diastolic flow velocity (Figure 6). The Doppler flow velocities suggested that some glaucomatous subjects had slow flow velocities while others were in the range of the normals. In the NTG group there were many patients with a slower flow velocity compared with the range of the normals.

There is a possibility that systemic or topical medications used by the glaucoma patient may be responsible for the reduction of ophthalmic artery blood flow velocities. Details of medication at the time of test were available in 39 of 42 NTG patients. We compared the flow velocities of the 12 NTG patients who were on topical antiglaucoma medication with or without systemic

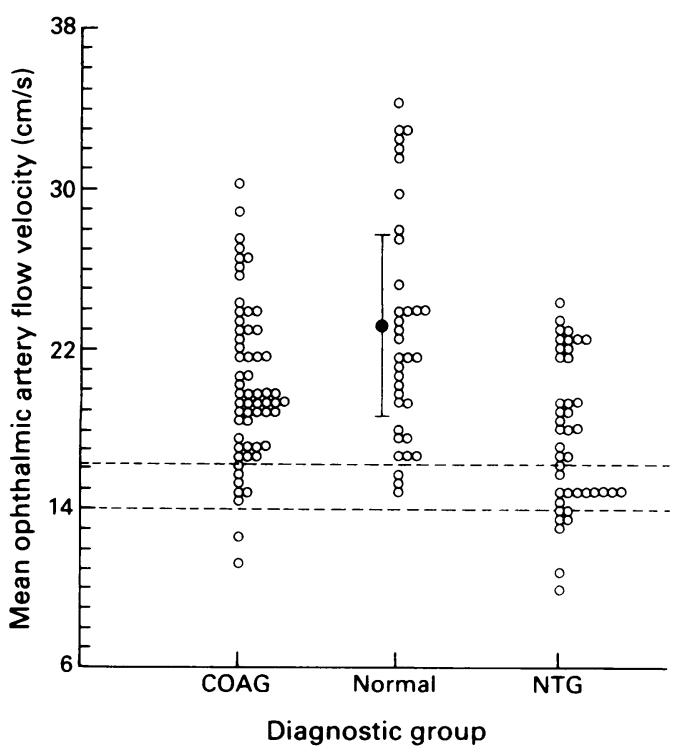

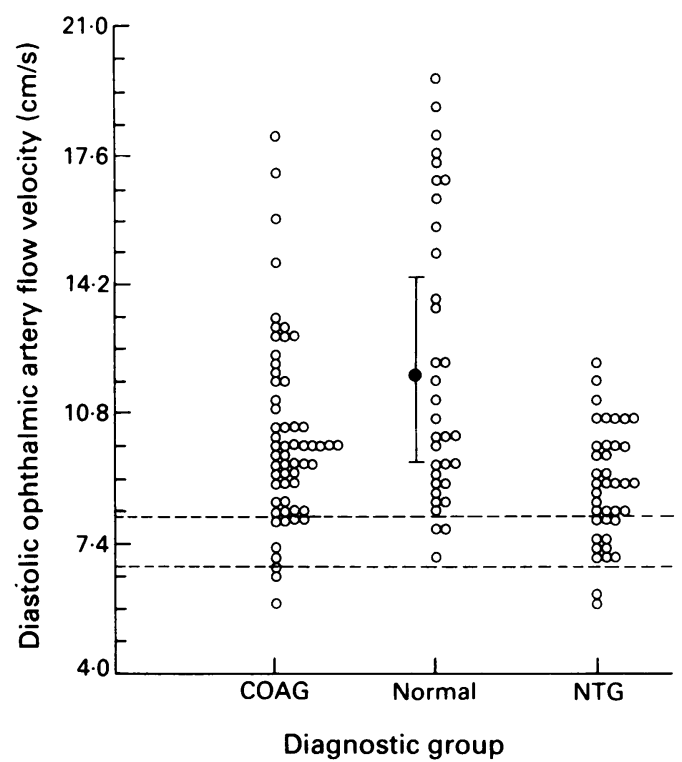

Figure 3 A dot density plot of the right ophthalmic artery diastolic flow velocity $(\mathrm{cm} / \mathrm{s})$ of 59 chronic open angle glaucoma patients, 34 normal tension glaucoma, and 34 normals. Each dot represents the velocity of an eye. The means and standard deviations for the normals are shown: 1.5 and 2.0 standard deviations $(8.09$ and $6.82 \mathrm{~cm} / \mathrm{s}$ respectively) were marked by dotted lines.

anti-hypertensive agents with 27 NTG patients who were not on any medication, and found no statistically significant differences of any of the ophthalmic artery flow velocities (peak flow velocity $=35.65 \mathrm{~cm} / \mathrm{s}(8.45)$ vs $35.53 \mathrm{~cm} / \mathrm{s}(9.57)$; mean flow velocity $=18.02 \mathrm{~cm} / \mathrm{s}(3.67)$ vs 17.55 $\mathrm{cm} / \mathrm{s}(3.85)$ and diastolic flow velocity $=8.81$ $\mathrm{cm} / \mathrm{s}(1.61)$ vs $9.16 \mathrm{~cm} / \mathrm{s}(1.40)$, respectively; all p values $>0 \cdot 5)$.

\section{Discussion}

The pathogenesis of open angle glaucoma has not yet been fully worked out. Two main mechanisms have been considered. One suggests mechanical damage directly to the axons or small blood vessels $^{15}$ by structural alterations at the lamina cribrosa. The other suggests a primary problem in the optic nerve circulation as a result of localised organic changes ${ }^{16}$ in the blood vessels of the nerve with or without a low perfusion pressure. ${ }^{17}$ It is likely that both of these mechanisms may be involved in the development of the glaucomatous process either independently or influencing one another. There may be more

Figure 2 A dot density plot of the right ophthalmic artery mean flow velocity $(\mathrm{cm} / \mathrm{s})$ of 59 chronic open angle glaucoma patients, 42 normal tension glaucoma, and 35 normals. Each dot represents the velocity of an eye. The mean and standard deviations for the normals are shown: $1 \cdot 5$ and $2 \cdot 0$ standard deviations $(16 \cdot 26$ and $14.06 \mathrm{~cm} / \mathrm{s}$ respectively) were marked by dotted lines.

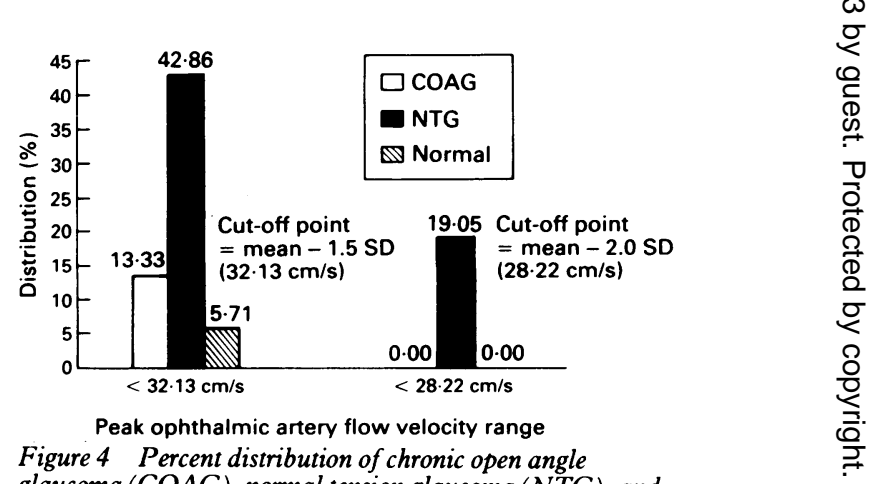
glaucoma (COAG), normal tension glaucoma (NTG), and normal subjects whose ophthalmic artery peak flow velocities were 1.5 standard deviation (left side) and 2.0 standard deviation (right side) lower than the mean of the normal. The percentages are labelled at the top of each bar. 
Figure 5 Percent distribution of chronic open angle glaucoma (COAG), normal tension glaucoma (NTG), and normal subjects whose ophthalmic artery mean flow velocity were 1.5 standard deviation (left side) and $2 \cdot 0$ standard deviation (right side) lower than the mean of the normal. The percentages are labelled at the top of each bar.

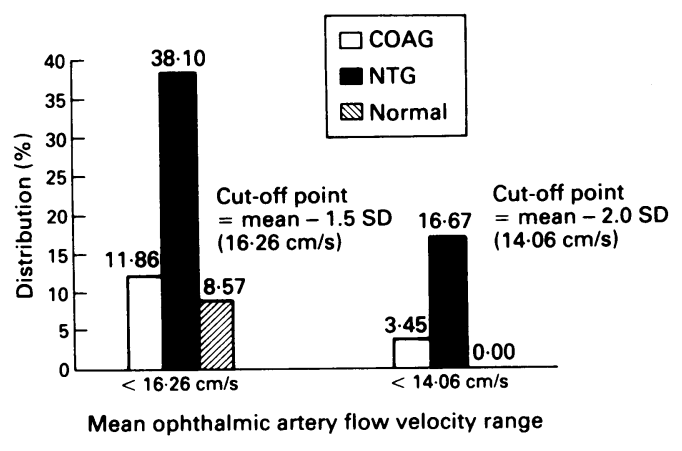

than one glaucomatous population and more than one mechanism of damage might be involved.

The advent of transcranial Doppler ultrasound, which allows the measurement of the blood flow velocity in the ophthalmic artery, was the impetus for comparing the ophthalmic flow velocity of normal humans with patients suffering from chronic open angle glaucoma and normal tension glaucoma. We found a significantly slower ophthalmic flow velocity in the NTG patients compared with our normals. The mean velocities of the COAG group were also significantly slower than the normals but were between the normals and the NTG patients. Our findings are similar to studies of the nailfold capillaries recently reported by Gasser and Flammer. ${ }^{18} \mathrm{~A}$ previous study of ophthalmic flow velocity in chronic open angle glaucoma and normals reported similar differences, but a different Doppler frequency $(8 \mathrm{MHz})$ was used so that all the values were approximately three times smaller than our results suggest. ${ }^{19}$ Using a $4 \mathrm{MHz}$ pulsed Doppler ultrasound in similar fashion to those which we employed showed velocities of normal subjects closer to ours. ${ }^{20}$ With duplex scanner $^{21}$ which employed $5 \mathrm{MHz}$ Doppler frequency and B scan imaging and another study using a colour Doppler system $7 \cdot 5 \mathrm{MHz}^{22}$ in normal subjects ( $n=72$ and 40 respectively), the peak ophthalmic flow velocities reported were closer to but still lower than those we obtained with the TCD system. The differences in normal peak flow values may be due to different groups of patients, their age, and most probably the different ultrasound systems. Comparing mean flow velocity in our normal group to another study which reported mean flow velocity in 106 normal subjects using TCD $2 \mathrm{MHz}$, showed our results to be similar to theirs ${ }^{11}$ (mean (SD) $22 \cdot 87$ $(5 \cdot 69)$ and 21 (5) respectively). With the TCD, it is possible to measure the Doppler shift from a more posterior part of the ophthalmic artery which may minimise the shift in Doppler angle

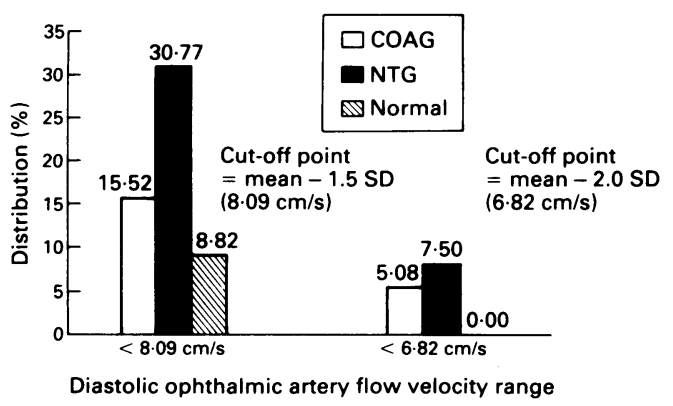

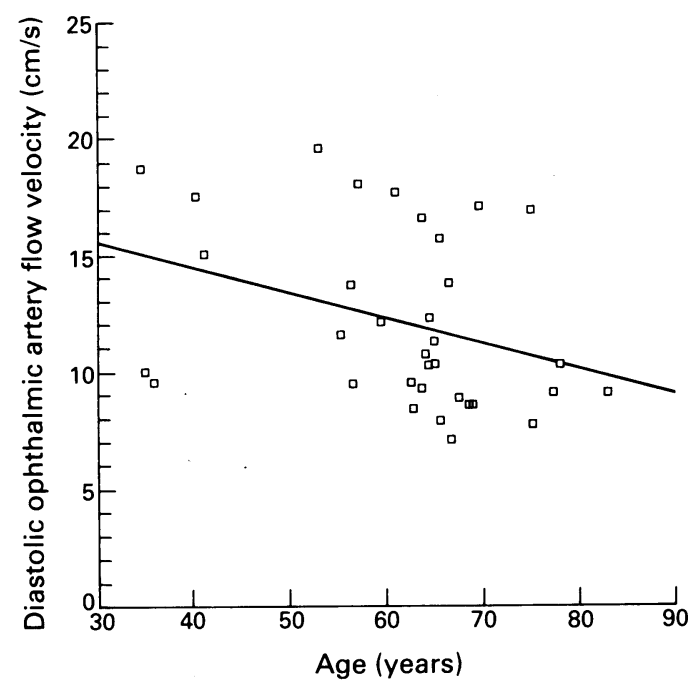

Figure 7 A scatter plot of diastolic flow velocity in $\mathrm{cm} / \mathrm{s}$ against age in normals showing age-related loss in flow velocity (standard coefficient $=0.355 p=0 \cdot 039$ ).

as the course of the artery is less variable there, and there is also less interference with the quality of the tracing. One can always be certain that one is recording the ophthalmic artery by its high resistance characteristics which are quite different from those produced by the carotid artery. The lack of image guidance for locating the ophthalmic artery makes it necessary to use the criteria described in our method.

The present study included only a relatively small normal population and did not include the younger age groups. In spite of this we were able to show that there was an age-related loss of ophthalmic flow velocities (Figure 7) but only the diastolic flow velocity showed a statistically significant correlation with age $(p=0.020)$, the probabilities of the correlations for the peak and mean velocities were 0.061 and 0.114 respectively. In a larger population of normal subjects which included younger subjects, we will be reporting a negative correlation between age and all three ophthalmic flow velocities. This association was also found by others in normal subjects ${ }^{1521}$ and also in a carotid Doppler study. ${ }^{23}$ Our normal group had a slightly higher proportion of males than the NTG group but less than the COAG group but as we found no statistically significant difference of the ophthalmic artery flow velocity between males and females except for the diastolic flow velocity in the normal group, the sex difference is not likely to be responsible for the results. The blood pressure and the pulse rate were not significantly different between the three groups. We found no difference in all three flow velocities between the NTG patients who were on anti-glaucoma medication with or without systemic medications and those who were not, so the medication is not likely to be responsible for our results. It is possible that the significant differences between the three groups could be due to the accumulation of small differences in the other variables such as systolic and diastolic blood pressure, pulse rate, and sex ratio. However for this to be true there would have to be a strong negative association between the flow velocity measurements and blood pressure value, a strong positive association between pulse rate and the flow velocities, and side) and $2 \cdot 0$ standard deviation (right side) lower than the mean of the normal. The percentages are labelled at the top of each bar. 
being female would have to be associated with lower peak and mean flow as well as diastolic flow, which was not the case.

The cause and significance of the reduction of ophthalmic flow velocity in some glaucoma patients has yet to be elucidated. Whether the differences in the flow velocity between some glaucoma patients and their normal counterparts play any role in the pathogenesis of the glaucomas is unknown. There were individuals in the glaucoma groups who had marked reductions in their ophthalmic flow velocity whereas others appeared to have a normal flow. The study of some other variables such as intraocular pressure, visual field defects, and optic disc topography in conjunction with the ophthalmic artery flow velocity may provide interesting results. If accurate measurement of the diameter of the ophthalmic artery were possible by an imaging system coupled with the TCD ultrasound, this would allow studies of the blood flow as opposed to the flow velocity in the ophthalmic artery and might shed more light on the haemodynamics of the ocular blood flow. Future colour flow imaging ultrasound may achieve this goal.

1 Becker B. Diabetes mellitus and primary open-angle glaucoma. Am f Ophthalmol 1971; 71: 1-16.

2 Mcleod SD, West SK, Quigley HA, Fozard JL. A longitudinal study of the relationship between intraocular and blood pressures. Invest Ophthalmol Vis Sci 1990; 31: 2361-6.

3 Phelps CD, Corbett JJ. Migraine and low-tension glaucoma. A case-control study. Invest Ophthalmol Vis Sci 1985; 26: 1105-8.

4 Drance SM, Douglas GR, Wijsman K, Schulzer M, Britton RJ. Response of blood flow to warm and cold in normal and low-tension glaucoma patients. Am $\mathcal{F}$ Ophthalmol 1988; 105: 35-9.

5 Flammer J, Guthauser U, Mahler M. Do ocular vasospasms help cause low-tension glaucoma? Doc Ophthamol Proc Ser 1987; 397: 397-9.

6 Gasser P. Ocular vasospasm, a risk factor in the pathogenesis of low-tension glaucoma. Int Ophthalmol 1989; 13: 281-90.
7 Drance SM, Sweeney VP, Morgan RW, Feldman F. Studies of factors involved in the production of low tension glaucoma Arch Ophthalmol 1973; 89: 457-65.

8 Carter CJ, Brooks DE, Doyle DL, Drance SM. Investigations into a vascular etiology for low-tension glaucoma. Ophthalmology 1990; 97: 49-55.

9 Spencer MP, Hileman RE, Reid JM. Ultrasound physical concepts. In: Spencer MP, ed. Ultrasonic diagnosis of cerebrovascular disease. Dordrecht: Martinus Nijhoff, 1987: $19,21,25$.

10 Aaslid R, Markwalder TM, Nornes H. Noninvasive transcranial Doppler ultrasound recording of flow velocity in basal cerebral arteries. F Neurosurg 1982; 57: 769-41.

11 Ringelstein EB. A practical guide to transcranial Doppler sonography. In: Weinberger J, ed. Noninvasive imaging of cerebrovascular disease. New York: Alan R Liss 1989: Chap $5,80-1$.

12 Rojanapongpun P, Morrison BJ, Drance SM. Ophthalmic artery flow velocity by transcranial Doppler ultrasound; reproducibility and potential application in ophthalmology. BrF Ophthalmol 1993; 77: 22-24.

13 Fish P. Physics and instrumentation of diagnostic medical ultrasound. Chichester: Wiley, 1990: 218.

14 Wilkinson L. The system for statistics. Evanston, IL: Systat, 1990: 261 .

15 Quigley HA, Addick EM, Green WR, Maumenee AE. Optic nerve damage in glaucoma. Arch Ophthalmol 1981; 99: 6359.

16 Hayreh SS. Blood supply of the optic nerve head and its role in optic atrophy, glaucoma and papilloedema. BrF Ophthalmol 1969; 53: $721-48$.

17 Hayreh SS. Interindividual variation in blood supply of the optic nerve head. Its importance in various ischemic disorders of the optic nerve head and glaucoma, LTG and allied disorders. Doc Ophthalmol 1985; 59: 217-46.

18 Gasser P, Flammer J. Blood-cell velocity in the nailfold capillaries of patients with normal-tension glaucoma. $A m \mathcal{F}$ Ophthalmol 1991; 111: 585-8.

19 Martorina M, Camerlingo M. A Doppler-sonographic study in glaucoma. Ophthalmologica 1987; 194: 82-5.

20 Michelson G, Grierth K, Priem R, Laumer R. Blutflußgeschwindigkeit in der A. ophthalmica durch transbulbare Dopplersonographie. Fortschr Ophthalmol 1989; 86: 3313.

21 Guthoff RF, Berger RW, Winkler P, Helmke K, Chumbley LC. Doppler ultrasonography of the ophthalmic and central retinal vessels. Arch Ophthalmol 1991; 109: 532-6.

22 Lieb WE, Cohen SM, Merton DA, Shields JA, Mitchell DG, Goldberg BB. Color Doppler imaging of the eye and orbit. Technique and normal vascular anatomy. Arch Ophthalmol 1991; 109: 527-31.

23 Fabris F, Poli L, Zanocchi M, Govone G. Age-related modifications of carotid Doppler findings. In: Crepaldi $G$ modifications of carotid Doppler findings. In: Crepaldi G, New York: Raven Press, 1983: 91-4. 